\title{
Supporting Information \\ Penetration Behavior of a Water Droplet into a Cylindrical Hydrophobic Pore
}

\author{
Yoshimune Nonomura, TomoyaTanaka, and Hiroyuki Mayama
}

\section{Derivations of factors.}

Fig. S1 illustrates schematic representations of the spreading, penetration and breaking patterns. Figs. S1a shows the spreading pattern. The droplet does not penetrate into the pore after falling. Figs. S1b and c are the penetration pattern, but their situations are different. Under the condition of $D_{p}<D_{d}$, the former is observed after falling. Under $D_{p} \leq D_{d}$, the latter is observed without falling. In other words, it is accomplished when we put a droplet over a pore, i.e., spontaneous penetration. Thus, we found that there are two types in the penetration pattern. Fig. S1d shows the breaking pattern. The initial state is the penetration pattern. The droplet is divided after falling. One part remains, other part remains as a divided liquid film, or a droplet on wall and substrate (see, also Fig. S2h).

To understand the actual penetration phenomena accomplished by falling, we mention the balance between driving and suppression factors as shown in Fig. S2. There are eight factors. The driving factors are potential energy of a droplet $\Delta U$ (Figs. S2a), potential energy of a droplet by finite radius $\Delta U_{\text {radius }}$ (Fig. S2b), and potential energy change of a droplet over a pore by gravity $E_{\text {grav }}$ (Fig. S2c). On the other hand, the suppression factors are work to move liquid as a cylinder into pore after falling $W_{\text {cylinder }}$ (Fig. S2d), pinning energy at the edge of a pore $E_{\text {pin }}$ (Fig. S2e), change in surface energy by the change in shape from a sphere to a cylinder $\Delta E_{\text {surf }}$ (Fig. S2f), energy dissipation due to viscosity $E_{d i s}$ (Fig. S2g), breaking energy $E_{\text {break }}$ (Fig. S2h). Here, $\Delta U_{\text {grav }}$ and $E_{\text {pin }}$ can be neglected in the experimental results $\left(D_{p} / D_{d}>0.34\right)$ as discussed later.

\section{1-1. Potential energy converted into kinetic energy (Fig. S2a)}

We first discuss the driving force. The change $\Delta U$ in potential energy of a droplet dropped from height $h$ is 


$$
\Delta U=m g h=\frac{4 \pi}{3} \rho\left(\frac{D_{d}}{2}\right)^{3} g h=\frac{\pi}{6} D_{d}^{3} \rho g h .
$$

Here, $h$ is defined as the distance between the bottom of droplet and substrate, This is one of the driving factors.

\section{1-2. Potential energy of droplet due to finite radius (Fig. S2b)}

The size of droplet should be considered as potential energy because it is comparable to small $h$ the in the present experiments. In other words, the droplet over substrate has potential energy itself due to its finite radius. Potential energy due to the radius $\Delta U_{\text {radius }}$ is

$$
\Delta U_{\text {radius }}=\frac{\pi}{6} \rho g D_{d}^{3}\left(\frac{D_{d}}{2}\right) .
$$

This works as a driving factor.

\section{1-3. Effect of gravity on energy (Fig. S1c)}

Next, we discuss the effect of gravity on the initial condition. The droplet placed over the hole has a flat air-liquid interface; however, because of gravity, the air-liquid interface protrudes somewhat into the hole. The height of the center of mass is thus lowered by gravity. Its energy $E_{g r v}$ is

$$
E_{\text {grv }}=m g \Delta h,
$$

where $m$ is the mass of a droplet, $g$ is the gravitational acceleration, and $\Delta h$ is the decrease in the height of center of mass due to the protrusion into the hole.

Note that the protrusion depth of the air-liquid interface $d_{d i p}$ roughly equals $\Delta h$ if $D_{p} / D_{d}$ is close to unity. Therefore, to discuss $E_{g r v}$, we consider $d_{d i p}$ based on Laplace pressure $\Delta P$, which is generated by the weight of the droplet. The Laplace pressure is given by

$$
\Delta P=\frac{m g}{\pi\left(D_{p} / 2\right)^{2}}
$$

where the mass $m$ on the hole is

$$
m=\frac{4}{3} \pi\left(\frac{D_{d}}{2}\right)^{3} \rho \times\left(\frac{D_{p}}{D_{d}}\right)^{2}=\frac{\pi}{6} \rho D_{d} D_{p}^{2},
$$

where the factor $\left(D_{p} / D_{d}\right)^{2}$ is the ratio of the cross-sectional area to the droplet 
cross-sectional area, which allows us to introduce an effective mass for the hole. As a result, we obtain

$$
\Delta P=\frac{2}{3} \rho g D_{d}
$$

However, this results is directly related to $\gamma_{L}$ and to the radius of curvature $r$ by

$$
\Delta P=\frac{2 \gamma_{L}}{r}
$$

and

$$
r=\frac{D_{p}}{2 \sin \varphi}
$$

The dipping depth of the air-liquid interface $d_{d i p}$ is obtained from the geometry given in Figure S1(b):

$$
d_{d i p}=r(1-\cos \varphi) \sim \Delta h .
$$

As a result, we get the following relationship from Eqs. (S2)-(S4):

$$
r=\frac{3 \gamma_{L}}{\rho g D_{d}}
$$

and $\varphi$ as

$$
\varphi=\sin ^{-1}\left(\frac{\rho g D_{d} D_{p}}{6 \gamma_{L}}\right)
$$

Equation (S10) says that the interface is dipped if $D_{d}$ and/or $D_{p}$ increase. We thus obtain

$$
E_{g r v}=\frac{\pi}{2} \gamma_{L} D_{d}^{2}(1-\cos \varphi)
$$

$E_{g r v}$ is a driving factor. However, $E_{g r v}$ is effective in the conditions of $D_{p} / D_{d}<$ 0.34 as discussed in 1-5 Pinning energy. Actually, $E_{g r v}$ can be neglected in our experiments $\left(D_{p} / D_{d}>0.56\right)$.

\section{1-4. Work to move liquid as a cylindrical droplet into a pore (Fig. S2d)}

After penetration, the droplet stays in pore and its shape changes from a sphere to a cylinder. However, the center of mass should be moved. Work to move the cylinder $W_{\text {cylinder }}$ is

$$
W_{\text {cylinder }}=\frac{\pi}{6} \rho g D_{d}^{3}\left(\frac{d}{2}\right),
$$

where $d$ is the thickness of the cylinder. $W_{\text {cylinder }}$ works as a suppression factor. 


\section{1-5. Pinning energy (Fig. S2e)}

Next, we discuss $E_{\text {pin }}$ based on the Laplace pressure $\Delta P$ and work to push the droplet into the hole. There are two main reasons for the pinning effect: the first reason is the presences of physical defects such as sharp edges and the second reason is the presence of chemical defects such as contamination. In our case, the sharp edge of the hole pins the contact line and serves as a physical defect. Figure S1 shows schematic representations of a droplet on a hydrophobic hole before and after pressure is applied. To dip the air-liquid interface in the hole, we apply pressure (the Laplace pressure) onto the interface indicated by the arrow in Figure S1(a). If we increase the pressure, the interface dips into the hole and moves, as shown in Figure S1(b). If the dipping angle $\varphi$ reaches the critical dipping angle $\varphi_{c}$, the droplet penetrates into the hole, as shown in Figure S1(c). Therefore, the work done by the Laplace pressure on to the interface may be obtained from the applied force and the displacement of the interface. We assume that the penetration occurs if the dipping angle reaches the critical dipping angle. As a result, the work required for the penetration may be calculated based on $\Delta P$, which is

$$
\Delta P=\frac{2 \gamma_{L}}{r}
$$

Since force is the product of area and pressure, the surface area of the air-liquid interface in the hole, $A_{\text {in-hole}}$, which corresponds to $A_{L 2}$ in our experiments, should be considered. This is

$$
A_{\text {in-hole }}=2 \pi r^{2}(1-\cos \varphi),
$$

where $\varphi$ is dipping angle. To describe $A_{\text {in-hole }}=\pi\left(D_{p} / 2\right)^{2}$ at $\varphi=0$ consistently, Eq. (3) is modified as

$$
A_{\text {in-hole }}=\frac{2 \pi\left(D_{p} / 2\right)^{2}}{1+\cos \varphi} .
$$

Here, we adopt $r=\left(D_{p} / 2\right) / \sin \varphi$. Force applied on the interface $F$ is

$$
F=\Delta P \times A_{\text {in-hole }}=\frac{2 \gamma_{L}}{R} \times \frac{2 \pi\left(D_{p} / 2\right)^{2}}{1+\cos \varphi}=\frac{2 \gamma_{L} \sin \varphi}{\left(D_{p} / 2\right)} \times \frac{2 \pi\left(D_{p} / 2\right)^{2}}{1+\cos \varphi}=\frac{4 \pi \gamma_{L}\left(D_{p} / 2\right) \sin \varphi}{1+\cos \varphi} \propto D_{p} .
$$

Since work $W$ is the product of force $F$ and displacement $d x, d x$ should be considered. 
This is

$$
d x=\frac{D_{p}}{2} d \varphi
$$

Therefore, $W$ is

$$
W=\int_{0}^{x} F d x=\int_{0}^{\varphi_{\mathrm{c}}} \frac{4 \pi \gamma_{L}\left(D_{p} / 2\right) \sin \varphi}{1+\cos \varphi} \frac{D_{p}}{2} d \varphi=4 \pi \gamma_{L}\left(\frac{D_{p}}{2}\right)^{2} \int_{0}^{\varphi_{\mathrm{c}}} \frac{\sin \varphi}{1+\cos \varphi} d \varphi .
$$

where $\varphi_{c}$ is the critical dipping angle (discussed below). We use integration by substitution to do the integration in Eq. (7). If we use $t=\cos \varphi$, we obtain

$$
W=-4 \pi \gamma_{L}\left(\frac{D_{p}}{2}\right)^{2} \int_{t_{1}}^{t_{2}} \frac{d t}{1+t}=\pi \gamma_{L}\left[\ln \left(1+t_{1}\right)-\ln \left(1+t_{2}\right)\right] D_{p}^{2} \propto D_{p}^{2}
$$

where $t_{1}=1$ and $t_{2}=\cos \varphi_{c} . \quad W$ in Eq. (8) is equal to pinning energy $E_{\text {pin }} . E_{\text {pin }}$ is thus proportional to $D_{p}^{2}$ :

$$
E_{\text {pin }}=f_{\text {pin }}\left(\varphi_{c}\right) D_{p}^{2} \propto D_{p}^{2}
$$

where $f_{\text {pin }}\left(\varphi_{c}\right)$ is a function of the critical dipping angle $\varphi_{c}$ into the hole [Figure 1(d)]. Specifically,

$$
f_{\text {pin }}\left(\varphi_{c}\right)=\pi \gamma_{L}\left[\ln 2-\ln \left(1+\cos \varphi_{c}\right)\right]
$$

where

$$
\varphi_{c}=\theta_{e q}-\frac{\pi}{2}
$$

Note that $\varphi_{\mathrm{c}}$ is related to the equilibrium contact angle $\theta_{\mathrm{eq}}$ on flat hydrophobic surfaces $(>\pi / 2)$. In our experiments, $\theta_{e q}=110^{\circ}$. Note also that the pinning force and pinning energy are proportional to $D_{p}$ and $D_{p}{ }^{2}$, respectively. $E_{p i n}$ works as a suppression factor.

Now, we would like to show that $E_{\text {pin }}$ and $E_{\text {grav }}$ work in the conditions of $D_{p} / D_{d} \leq 0.34$. Figure S3 shows a geometry of a sphere contacting with the edge of pore. The relationship between $\varphi, D_{p}$ and $D_{d}$ is

$$
\sin \varphi=\frac{D_{p} / 2}{D_{d} / 2}=\frac{D_{p}}{D_{d}}
$$

Assuming $\varphi_{\mathrm{c}}=20^{\circ}$ roughly, $E_{\text {pin }}$ and $E_{\text {grav }}$ work is

$$
\frac{D_{p}}{D_{d}}<0.34
$$

Actually, $E_{\text {pin }}$ and $E_{\text {grav }}$ can be neglected in the present conditions $\left(D_{p} / D_{d} \geq 0.56\right)$. 


\section{1-6. Change in surface energy (Fig. S2f)}

The shape of a droplet changes from a sphere to a cylinder through whole process. Therefore, the change in surface energy $\Delta E_{\text {surf }}$ should be considered. Roughly, $\Delta E_{\text {surf }}$ can be estimated to be

$$
\Delta E_{\text {surf }}=\gamma_{L}\left(2 \times \pi\left(\frac{D_{p}}{2}\right)^{2}+\pi D_{p} d\right)-4 \pi \gamma_{L}\left(\frac{D_{d}}{2}\right)^{2} .
$$

$\Delta E_{\text {surf }}$ works as a suppression factor.

\section{1-7. Dissipation energy (Fig. S2g)}

Next, we discus $E_{d i s}$. According to fluid mechanics, the rate of energy dissipation of a fluid is

$$
T \dot{S}=T \int\left[\frac{\kappa(\operatorname{grad} T)^{2}}{T^{2}}+\frac{\eta}{2 T}\left(\frac{\partial v_{i}}{\partial x_{k}}+\frac{\partial v_{k}}{\partial x_{i}}-\frac{2}{3} \delta_{i k} \frac{\partial v_{l}}{\partial x_{l}}\right)^{2}+\frac{\zeta}{T}(\operatorname{div} \vec{v})^{2}\right] d V,
$$

Where $T$ is the absolute temperature; $S$ is the entropy; $\kappa, \eta$, and $\zeta$ are the thermal conductivity and the usual first and second viscosity coefficients, respectively; $v_{i}, v_{k}$, and $v_{l}$ are the tensor components for velocity; $x_{i}, x_{k}$, and $x_{l}$ are the tensor components for the coordinating axes; $\delta_{i k}$ is the Kronecker delta; and $\vec{v}$ is the fluid velocity [Landau, L. D.; Lifshitz, E. M. Fluid Mechanics, 2nd ed.; Butterworth-Heinemann; Boston, 1998; p. 195]. For a thermally isotropic system with an incompressible fluid and using cylindrical coordinates, we get

$$
T \dot{S}=\int\left[\frac{\eta}{2}\left(\frac{\partial v_{i}}{\partial x_{k}}+\frac{\partial v_{k}}{\partial x_{i}}-\frac{2}{3} \delta_{i k} \frac{\partial v_{l}}{\partial x_{l}}\right)^{2}\right] d V .
$$

Therefore, $E_{d i s}$ can be obtained by integrating $T \dot{S}$ :

$$
E_{\text {dis }}=\int_{0}^{t} T \dot{S} d t
$$

We adopt cylindrical coordinates to describe the penetration of a droplet into a hole. Since the penetration occurs along the $z$ direction and $x=y$, from Eq. (S6) we get

$$
T \dot{S}=\int \eta\left(\frac{d v_{z}(x)}{d x}\right)^{2} d V .
$$

The next point is $d v_{z}(x) / \mathrm{d} x$, which is related to the velocity $v_{c}$ of the center of the droplet as

$$
\frac{d v_{z}(x)}{d x}=\frac{v_{c}}{R}
$$


where $x$ is the distance from the droplet edge to its center, $R=D_{p} / 2$, and $v_{c}$ is the fluid velocity at the center. Therefore,

$$
T \dot{S}=\int_{0}^{R} \int_{0}^{R} \int_{0}^{d} \eta\left(\frac{v_{c}}{R}\right)^{2} d x d y d z \approx \eta d v_{c}^{2},
$$

where $d$ is the thickness of droplet in pore. We now introduce the timescale of motion for the droplet $t_{m}$ to obtain $\mathrm{E}_{\mathrm{dis}}$ :

$$
E_{\text {dis }}=\int_{0}^{t_{m}} T \dot{S} d t \approx \eta d v_{c}^{2} t_{m}
$$

Since $v_{c}$ in free fall is

$$
v_{c}=\sqrt{2 g h} \text {. }
$$

We get

$$
E_{d i s} \approx 2 \eta d g h t_{m}
$$

$E_{d i s}$ works as a suppression factor. We assume that $t_{m}$ is the time for the first oscillation. Its order is estimated to be the order of $10 \mathrm{~ms}$ or less from Fig. 2. $E_{d i s}$ also plays a significant role in splitting process from the penetration and breaking patterns as discussed below.

\section{1-8. Breaking energy through splitting process (Fig. S2h)}

In the actual breaking pattern, we found that one droplets remain in the pore, and other also remains in pore as a liquid film, on wall or substrate as shown in Fig. S2f. It is valid to explain that the breaking pattern is generated through splitting process. Roughly, energy to induce splitting would be equal to the surface energy to peel off the lower water-solid interface. Assuming that the upper part of the cylinder (height: $d / 2$ ) remain and the water-solid interface of the lower part (height: $d / 2$ ) is peeled off, the splitting energy $E_{\text {break }}$ is equal to the peeled interfacial energy:

$$
E_{\text {break }}=\pi \gamma_{S L} D_{p} \frac{d}{2}
$$

$E_{\text {break }}$ works as a suppression factor in the breaking pattern. In the splitting process, $E_{\text {dis }}$ is also important because gradient of velocity is generated as shown in Fig. S4h.

\section{1-9. Short summary}

As mentioned above, we mention six effective factors in our conditions. $\Delta U$ and $\Delta U_{\text {radius }}$ are driving factors, whereas $W_{\text {cylinder }}, E_{\text {surf }}, E_{\text {dis }}$ and $E_{\text {break }}$ are 
suppression factors. The conditions in which factors become effective depend on the magnitude correlation between $D_{p}$ and $D_{d}$.

\section{Magnitude correlation between factors in spreading, penetration and breaking patterns.}

In this section, we discuss the criteria between the spreading pattern, the penetration patterns, and the breaking pattern.

\section{2-1. In spreading pattern (Fig. Sla)}

In the spreading pattern, the droplet still keeps its positon over the pore after falling. Therefore, the driving factors in magnitude must be smaller than that of the suppression factors:

$$
\Delta U+\Delta U_{\text {radius }}<W_{\text {cylinder }}+\Delta E_{\text {surf }}+E_{\text {dis }} .
$$

\section{2-2. In penetration pattern driven by $\Delta U$ (Fig. S1b)}

Under the situation of Fig. S1b, the penetration is achieved with energy dissipation because a droplet penetrates into a small pore. In the penetration pattern, the sum of the driving factors in magnitude must be larger than that of the suppression factors:

$$
\Delta U+\Delta U_{\text {radius }} \geq W_{\text {cylinder }}+\Delta E_{\text {surf }}+E_{\text {dis }} .
$$

\section{2-3. In penetration pattern by spontaneous penetration (Figs. S1c and S4)}

Spontaneous penetration occurs at $h \sim 0$ if $D_{p}$ is close to $D_{d}$. For simply, we roughly assume that driving factor is converted to $W_{\text {cylinder }}$ :

$$
\Delta U+\Delta U_{\text {radius }} \geq W_{\text {cylinder }} \text {. }
$$

We found that this explains the phase boundary between the spreading and penetration patterns at $h=0$ in the experimental results.

\section{2-4. In breaking pattern through splitting process (Figs. S1d and S2h)}

The breaking pattern always appears after the penetration pattern. If the wetting behavior before the breaking pattern could be taken into account for the spontaneous 
penetration, $E_{\text {break }}$ could be included as suppression factor by splitting process with energy dissipation in eq. (S39):

$$
\Delta U+\Delta U_{\text {radius }} \geq W_{\text {cylinder }}+E_{\text {dis }}+E_{\text {break }} \text {. }
$$

where the relation between $\Delta U, \Delta U_{\text {radius }}$ and $W_{\text {cylinder }}$ is given by eq. (S39). Therefore, $E_{\text {dis }}$ and $E_{\text {break }}$ are additional energies.

\section{Phase-border lines between spreading, penetration and breaking patterns.}

Based on the above discussion, there are three phase-border lines by eqs. (S38)-(S40). We describe the phase diagram of the penetration of a droplet into a pore as a function of $D_{d}, D_{p}$, and, $h$ to compare with Figure 3 although the theoretical criteria between the spreading, penetration and breaking patterns are different from the experimental criteria.

\section{3-1. Phase-border line between spreading and penetration patterns}

Actually, we found two kinds of phase-border lines between the spreading and penetration patterns. One is by eq. (S38) and other is by (S39) One is a penetration driven by $\Delta U$, other is a spontaneous penetration. First, we discuss the former case. From Eq. (S38), the critical condition is

$$
\Delta U+\Delta U_{\text {radius }}=W_{\text {cylinder }}+\Delta E_{\text {surf }}+E_{\text {dis }} \text {. }
$$

Using $D_{d}, D_{p}$, and, $h$ and Eqs. (S1), (S11), (S19), and (S28), we obtain

$$
\begin{gathered}
\frac{\pi}{6} D_{d}^{3} \rho g h+\frac{\pi}{6} D_{d}^{3} \rho g\left(\frac{D_{d}}{2}\right)=\frac{\pi}{6} D_{d}^{3} \rho g\left(\frac{d}{2}\right)+\gamma_{L}\left(2 \times \pi\left(\frac{D_{p}}{2}\right)^{2}+\pi D_{p} d\right) \\
-4 \pi \gamma_{L}\left(\frac{D_{d}}{2}\right)^{2}+2 \eta d g h t_{m} .
\end{gathered}
$$

We modify as

$$
\begin{array}{r}
\frac{\pi}{6} D_{d}^{3} \rho g\left(1-\frac{2 \eta d g t_{m}}{\frac{\pi}{6} D_{d}^{3} \rho g}\right) h=\frac{\pi}{6} D_{d}^{3} \rho g\left(\frac{d}{2}\right) \\
\gamma_{L}\left(2 \times \pi\left(\frac{D_{p}}{2}\right)^{2}+\pi D_{p} d\right) \\
-4 \pi \gamma_{L}\left(\frac{D_{d}}{2}\right)^{2}-\frac{\pi}{6} D_{d}^{3} \rho g\left(\frac{D_{d}}{2}\right) .
\end{array}
$$

As a result, we obtain the first phase-border line between spreading and penetration patterns $h_{s p 1}$ when $D_{p}<D_{d}$ and $h_{1}>D_{d}$ :

$$
h_{s p 1} \approx \frac{4 \gamma_{S L}}{\rho g D_{d}} \alpha^{-1}+\frac{2^{5} \gamma_{S L} \eta t_{m}}{\pi \rho^{2} g D_{d}^{3}} \alpha^{-3}+\left(\frac{3 \cdot 2^{5} \gamma_{L} \eta^{2} t_{m}^{2}}{\pi^{2} \rho^{3} g D_{d}^{5}}+\frac{D_{d}}{3}-\frac{3 \cdot 2^{4} \gamma_{L} \eta t_{m}}{\pi \rho^{2} g D_{d}^{3}}-\frac{2^{2} \eta t_{m}}{\pi \rho D_{d}}\right) \alpha^{-2}
$$




$$
\begin{aligned}
& +\left(\frac{2^{3} \eta t_{m}}{3 \pi \rho D_{d}}-\frac{3 \cdot 2^{7} \gamma_{L} \eta^{2} t_{m}^{2}}{\pi^{2} \rho^{3} g D_{d}^{5}}-\frac{2^{5} \eta^{2} t_{m}^{2}}{\pi \rho^{2} D_{d}^{3}}\right) \alpha^{-4}+\frac{2^{8} \gamma_{S L} \eta^{2} t_{m}^{2}}{\pi^{2} \rho^{3} g D_{d}^{5}} \alpha^{-5}+\frac{2^{6} \eta^{2} t_{m}^{2}}{3 \pi^{2} \rho^{2} D_{d}^{3}} \alpha^{-6} \\
& +\frac{3 \gamma_{L}}{\rho g D_{d}} \alpha^{2}+\frac{3 \cdot 2^{3} \gamma_{L} \eta t_{m}}{\pi \rho^{2} g D_{d}^{3}}-\frac{6 \gamma_{L}}{\rho g D_{d}}-\frac{D_{d}}{2},
\end{aligned}
$$

$h_{s p 1}$ can be obtained as the sum of inverse proportions with higher orders.

In (S44),

$$
\begin{aligned}
\alpha & =\frac{D_{p}}{D_{d}}, \\
\frac{d}{D_{d}} & =\frac{2}{3}\left(\frac{D_{d}}{D_{p}}\right)^{2},
\end{aligned}
$$

from the condition of constant volume.

$$
\pi\left(\frac{D_{p}}{2}\right)^{2} d=\frac{4}{3} \pi\left(\frac{D_{d}}{2}\right)^{3} .
$$

To develop from eq. (S43) to eq. (S44), we used the following approximation.

$$
\frac{1}{1+x} \approx 1-x+x^{2},|x|<1 \text {. }
$$

It should be noted that the condition of $|x|<1$ in eq. (S48) determines the order of $t_{m}$ as

$$
\frac{2 \eta d g t_{m}}{\frac{\pi}{6} D_{d}^{3} \rho g}<1
$$

Using eq. (44), we get

$$
t_{m}<\frac{\rho D_{p}^{2}}{2^{3} \eta}=\frac{\rho D_{d}^{2}}{2^{3} \eta} \alpha^{2} .
$$

It is difficult to determine $t_{m}$ experimentally. Therefore, we roughly estimate the order of $t_{m}$ is $10 \mathrm{~ms}$ or less from Fig. 2.

The second phase-border line between the spreading and penetration patterns is due to spontaneous penetration. From eqs. (S1), (S2), (S13) and (S39), we get

$$
\frac{\pi}{6} \rho g D_{d}^{3} h+\frac{\pi}{6} \rho g D_{d}^{3}\left(\frac{D_{d}}{2}\right)=\frac{\pi}{6} \rho g D_{d}^{3}\left(\frac{d}{2}\right),
$$

and

$$
h=\frac{D_{d}}{3} \alpha^{-2}-\frac{D_{d}}{2} .
$$

Since spontaneous penetration occurs at $h=0$, the critical condition of spontaneous penetration $\alpha_{c}$ can be obtained as 


$$
\alpha_{c}=\sqrt{\frac{2}{3}}=0.82 .
$$

Around $\alpha_{c}=0.82$, the second phase-border line between the spreading and penetration patterns $h_{s p 2}$ is

$$
h_{s p 2}=\frac{D_{d}}{3} \alpha^{-2}-\frac{D_{d}}{2} \text {. }
$$

As a result, $h_{s p 2}$ is obtained as a function of $\alpha^{-2}$ around $\alpha_{c}=0.82$.

\section{3-2. Phase-border line between penetration and breaking patterns}

From Eq. (S40), the critical condition separating the penetration and breaking patterns is

$$
\Delta U+\Delta U_{\text {radius }}=W_{\text {cylinder }}+E_{\text {dis }}+E_{\text {break }} .
$$

We modify this as

$$
\Delta U=W_{\text {cylinder }}-\Delta U_{\text {radius }}+E_{\text {dis }}+E_{\text {break }} .
$$

The relation between $\Delta U, \Delta U_{\text {radius }}$ and $W_{\text {cylinder }}$ is given by eq. (S55) because the wetting behavior before the breaking pattern could be taken into account for the spontaneous penetration. The phase-border line between the penetration and breaking patterns $h_{p b}$ is

$$
h_{p b}=h_{s p 2}+\Delta h \text {. }
$$

Here, $\Delta h$ is due to $E_{d i s}$ and $E_{\text {berak }}$. It is obtained as follows.

$$
\Delta U=E_{\text {dis }}+E_{\text {break }} .
$$

Using eqs. (S1), (S35) and (S36), eq. (S59) is modified as

$$
\frac{\pi}{6} D_{d}^{3} \rho g\left(1-\frac{2 \eta d g t_{m}}{\frac{\pi}{6} D_{d}^{3} \rho g}\right) \Delta h=\pi \gamma_{S L} D_{p} \frac{d}{2} .
$$

As a result, we get $\Delta h$ as

$$
\Delta h=\frac{2 \gamma_{S L}}{\rho g D_{d}} \alpha^{-1}+\frac{2^{4} \gamma_{S L} \eta t_{m}}{\pi \rho^{2} D_{d}^{3}} \alpha^{-3}+\frac{2^{6} \gamma_{S L} \eta^{2} t_{m}^{2}}{3 \pi^{2} \rho^{3} D_{d}^{5}} \alpha^{-5} .
$$

We assume that the order of $t_{m}$ in the breaking pattern is almost same to that in the penetration pattern. 


\section{Theoretical phase diagram}

Based on eqs. (S46), (S55) and (S58), theoretical phase diagrams are shown in Figure 5. Numerical parameters are summarized in Tab. S1. 


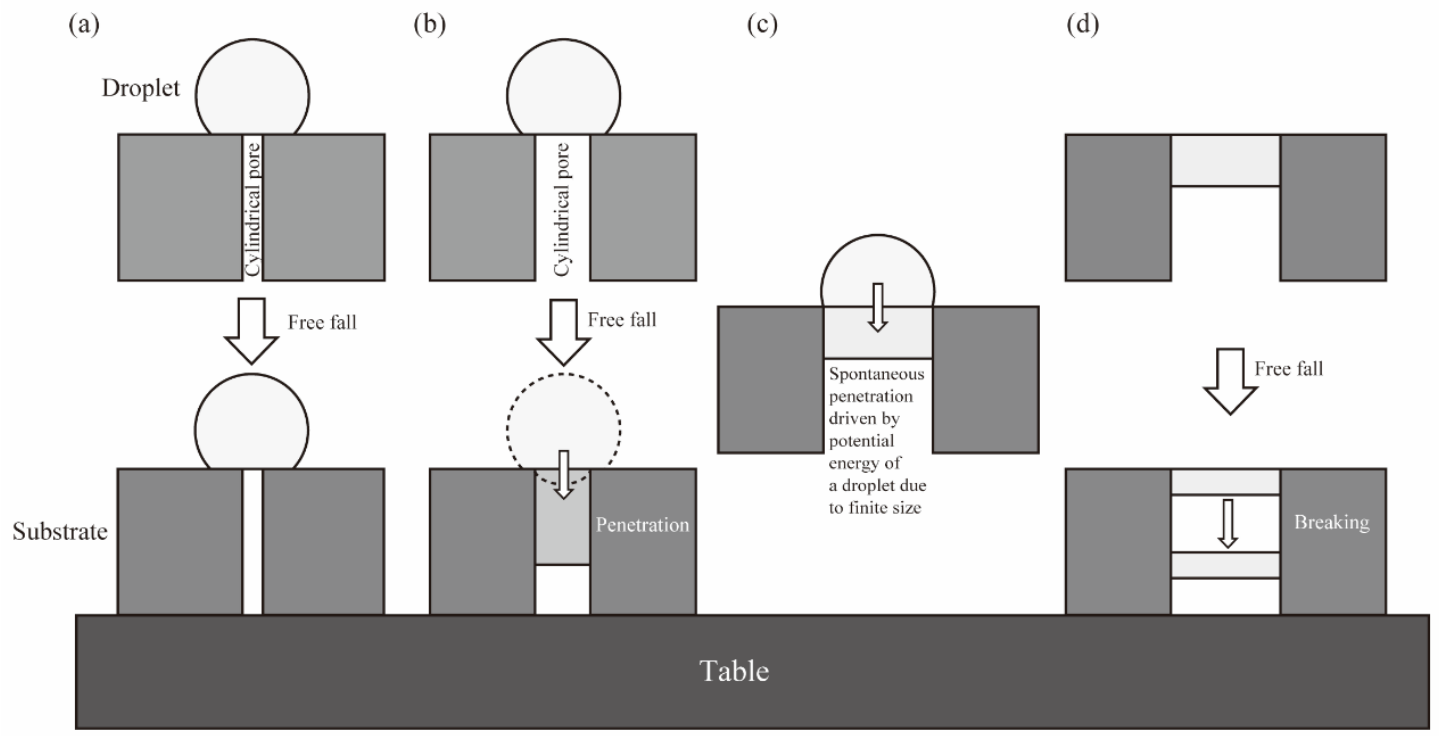

Figure S1. Schematic representations of typical behaviors under different conditions of $D_{p}$ and $D_{d}$. (a) Spreading pattern under $D_{p} \ll D_{d}$, (b) penetration pattern under $D_{p}<D_{d}$, (c) penetration pattern under $D_{p} \sim D_{d}$ by spontaneous penetration and (d) breaking pattern under $D_{p} \geq D_{d}$ 
(a) Potential energy

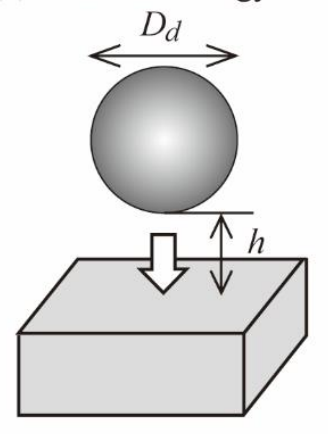

(b) Potential energy due to finite radius

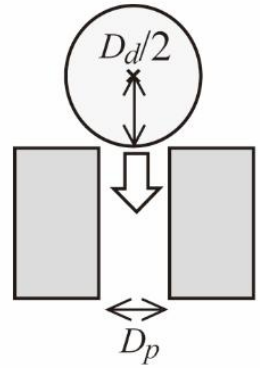

(c) Diping by gravity

$\left(D_{p} / D_{d}<0.34\right)$

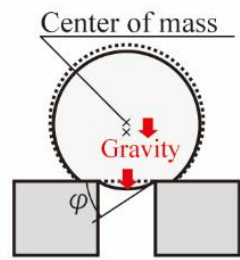

(d) Work to move a cylinder into a pore

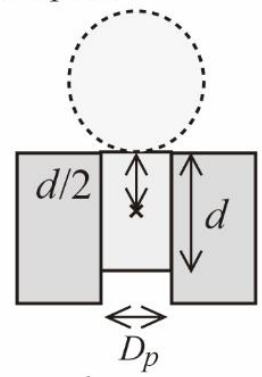

(f) Surface energy change

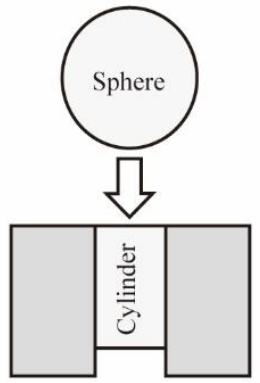

(g) Energy dissipation due to viscosity

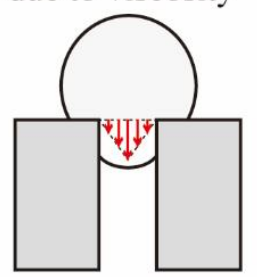

(e) Pinning at edge $\left(D_{p} / D_{d}<0.34\right)$

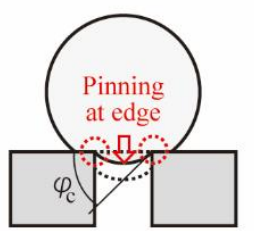

(h) Breaking through splitting process

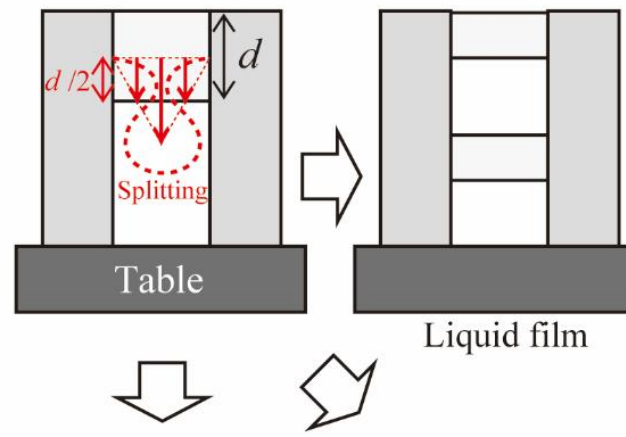

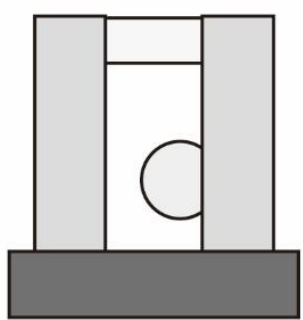

On wall

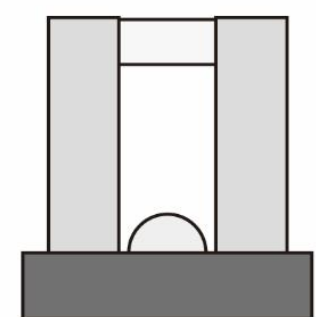

On substrate

Figure S2. Schematic representations of essential factors. (a) Potential energy at height $h$, (b) potential energy due to finite radius, (c) gravity effect, (d) work to move the droplet into the pore, (e) pinning energy at edge, (f) surface energy change, (g) energy dissipation due to viscosity, (h) breaking through splitting process with energy dissipation due to viscosity. 


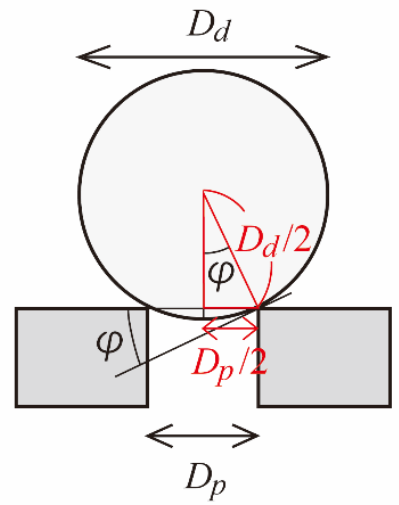

Figure S3. Geometry of a droplet on a pore and dipping angle 


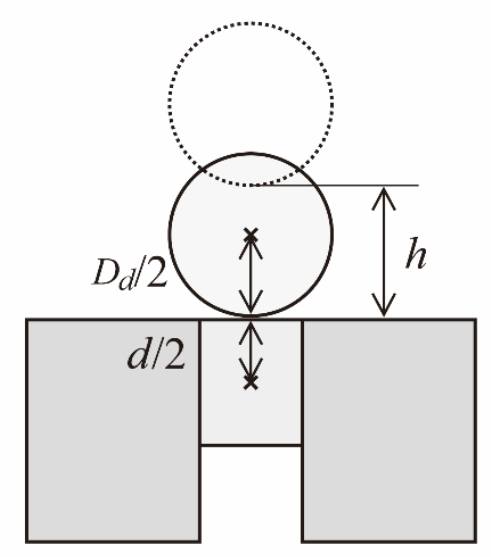

Figure S4. Schematic representation of Fig. S1c: a droplets over and in a pore under the conditions of $D_{d} \sim D_{p}$ and $h \sim D_{d}$. 
Table S1. Fitting parameters for theoretical phase-border lines between spreading penetration and penetration - breaking patterns. $D_{d}=1.79 \times 10^{-3} \mathrm{~m}$ and in all three conditions.

\begin{tabular}{|c|c|c|c|}
\hline & $\mathrm{H}_{2} \mathrm{O}$ & $20 \mathrm{wt} \%$ & $50 \mathrm{wt} \%$ \\
\hline$\gamma_{L}(\mathrm{mN} / \mathrm{m})$ & 72.8 & 72.4 & 70.0 \\
\hline$\gamma_{S L}(\mathrm{mN} / \mathrm{m})$ & 38.0 & 38.0 & 38.0 \\
\hline$\eta(\mathrm{Pa} \cdot \mathrm{s})$ & $0.89 \times 10^{-3}$ & $1.54 \times 10^{-3}$ & $5.04 \times 10^{-3}$ \\
\hline$\rho\left(\mathrm{g} / \mathrm{cm}^{3}\right)$ & 1.00 & 1.05 & 1.13 \\
\hline$t_{m}(\mathrm{~s})$ & 0.15 & 0.09 & 0.03 \\
\hline
\end{tabular}

